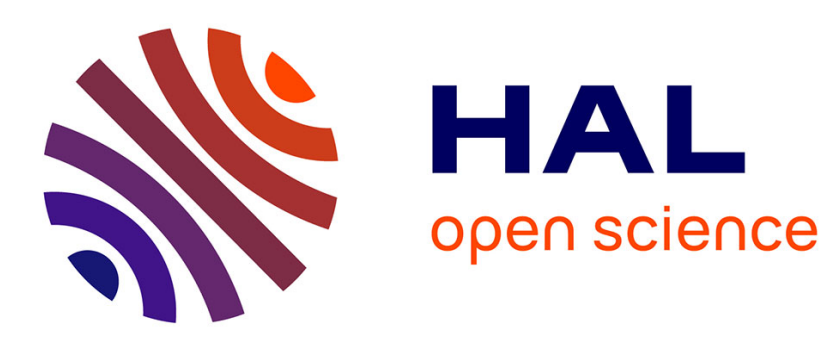

\title{
Derivation of Macroscopic Filtration Law for Transient Linear Viscoelastic Fluid Flow
}

B. Khuzhayorov, Jean-Louis Auriault, Pascale Royer

\section{To cite this version:}

B. Khuzhayorov, Jean-Louis Auriault, Pascale Royer. Derivation of Macroscopic Filtration Law for Transient Linear Viscoelastic Fluid Flow. International Journal of Engineering Science, 2000, 38 (5), pp.487-504. 10.1016/S0020-7225(99)00048-8 . hal-01714089

\author{
HAL Id: hal-01714089 \\ https://hal.science/hal-01714089
}

Submitted on 21 Feb 2018

HAL is a multi-disciplinary open access archive for the deposit and dissemination of scientific research documents, whether they are published or not. The documents may come from teaching and research institutions in France or abroad, or from public or private research centers.
L'archive ouverte pluridisciplinaire $\mathbf{H A L}$, est destinée au dépôt et à la diffusion de documents scientifiques de niveau recherche, publiés ou non, émanant des établissements d'enseignement et de recherche français ou étrangers, des laboratoires publics ou privés. 


\title{
Derivation of macroscopic filtration law for transient linear viscoelastic fluid flow in porous media
}

\author{
B. Khuzhayorov ${ }^{\text {a }}$, J.-L. Auriault ${ }^{\text {b }}$, P. Royer ${ }^{\text {b,* }}$ \\ ${ }^{a}$ Uzbekistan Academy of Sciences, Division of Samarkand, 3 Temur Malik Street, Samarkand City, Uzbekistan \\ b Laboratoire Sols, Solides, Structures, UJF, INPG, CNRS UMR 5521, BP 53X, 38041 Grenoble Cedex, France
}

\begin{abstract}
This work is concerned with deriving a macroscopic filtration law for describing transient linear viscoelastic fluid flow in porous media. This is performed using a homogenisation technique, i.e. by upscaling the heterogeneity scale description. The macroscopic filtration law is expressed in Fourier space as a generalised Darcy's law with a dynamic permeability tensor. This model is valid at low Reynolds and Deborah numbers. Analytical results are determined in the particular case of the flow of an Oldroyd fluid in a bundle of capillary tubes and are compared to those obtained by a corresponding phenomenological model.
\end{abstract}

Keywords: Homogenization; Filtration law; Linear viscoelastic fluid; Dynamic permeability

\section{Introduction}

Viscoelastic fluid flow in porous media is of interest for many engineering fields such as enhanced oil recovery, paper and textile coating, and composite manufacturing processes. The general linear viscoelastic model of fluid can describe some of the time-dependent motions of polymeric fluids, although it describes a restricted class of flows with very small displacement gradients.

Modelling of polymeric flow in porous media has motivated many studies that essentially focus on the numerical simulation of viscoelastic fluid flow in a specific pore geometry model, such as capillary tubes, indulating tubes, packs of spheres or cylinders. A good review of these studies can be found in [1]. Apparently, only a few mathematical macroscopic filtration models have been 
proposed concerning viscoelastic fluid flow in porous media. By analogy with Maxwell's model, the following phenomenological model has been introduced [2]:

$$
\vec{v}=-\frac{k}{\mu}\left(1+\lambda_{p} \frac{\partial}{\partial t}\right) \vec{\nabla} p .
$$

Then, on the basis of Oldroyd's model, the following filtration law for describing both relaxation and retardation phenomena was suggested [3]:

$$
\left(1+\lambda_{v} \frac{\partial}{\partial t}\right) \vec{v}=-\frac{k}{\mu}\left(1+\lambda_{p} \frac{\partial}{\partial t}\right) \overrightarrow{\nabla p}
$$

In this paper, we use the homogenisation theory for determining the macroscopic behaviour of viscoelastic fluid flow in porous media at low Reynolds and Deborah numbers. Homogenisation techniques allow determination of an equivalent macroscopic behaviour by upscaling the description at the heterogeneity scale. No specific geometry is at issue, the work is aimed towards deriving a general macroscopic mathematical model. Homogenisation method is introduced in Section 2. Section 3 sets out to derive the macroscopic behaviour of linear viscoelastic fluid flow in porous media. In Fourier space, the derived macroscopic filtration law is a generalised Darcy's law with a dynamic permeability $\tilde{K}(\omega)$. When inversed this law expresses a momentum balance in terms of a dynamic resistivity $\tilde{H}(\omega)=\tilde{K}^{-1}(\omega)$. This latter writing allows better physical interpretation as it shows how the fluid stress is related to both the viscous dissipation and the apparent density. Finally, in Section 4 the results in the particular case of the flow of an Oldroyd fluid in a bundle of capillary tubes are examined. It is shown that negative apparent density may occur. It turns out that the results obtained via homogenisation strongly differ from those provided by model (2).

\section{Introduction to homogenisation}

\subsection{Concept of homogenisability}

Modelling heterogeneous physical systems such as fluid flow in porous media turns out to be a difficult task. Nonetheless, the internal disorder may allow a large-scale continuous description. In other words, under specific conditions physical processes can be described by means of equations with transfer coefficients that are independent of the macroscopic boundary conditions. The essence of homogenisation techniques is to determine an equivalent macroscopic continuous behaviour by upscaling the description at the heterogeneity scale.

Homogenisation is possible if the density of heterogeneities is sufficiently high. For a material with a random structure, this intuitive condition is expressed by the existence of a Representative Elementary Volume (REV). In the context of a periodic medium, the REV is simply the period. The fundamental assumption behind homogenisation is the separation of scales, which can be expressed as follows: 


$$
l \ll L,
$$

where $l$ is the characteristic size of the REV and $L$ the characteristic macroscopic length. As this assumption conjures up a geometrical separation of scales, we shall draw attention to the fact that this fundamental condition must also be verified regarding the phenomenon. For instance, in the case of wave propagation, $l$ must also be small compared to the wavelength.

\subsection{Homogenisation for periodic structures}

\subsubsection{Fundamental assumptions}

In the present study, we use the method of homogenisation for periodic structures - also called method of multiple scales - introduced in $[4,5]$. As mentioned above, the fundamental assumption is the separation of scales and the key parameter of the method is the small parameter

$$
\varepsilon=\frac{l}{L} \ll 1,
$$

in which $L$ is the macroscopic characteristic length and, depending on the problem under consideration, is either geometrical (i.e. sample size) or related to the excitation (e.g. wavelength).

The medium is also assumed to be periodic; the period, denoted $\Omega$, is $\mathrm{O}(l)$. This assumption is actually not a restriction.

\subsubsection{Methodology}

We use the approach suggested in [6], by which the problem is tackled in a physical rather than mathematical manner. Indeed, it offers the additional benefit that conditions of homogenisability are expressly stated. This formulation of the method is on the basis of definition and estimation of the set of non-dimensional numbers arising from the description at the local scale. It allows determination of the macroscopic behaviour without any prerequisite on the form of the macroscopic equations.

2.2.2.1. Separation of space variables. As a result of the separation of scales, two non-dimensional space variables may be defined:

$$
\begin{aligned}
& \vec{y}=\frac{\vec{X}}{l} \quad \text { (non-dimensional microscopic space variable), } \\
& \vec{x}=\frac{\vec{X}}{L} \quad \text { (non-dimensional macroscopic space variable), }
\end{aligned}
$$

where $\vec{X}$ is the physical space variable.

If the condition of separation of scales is verified, then $\vec{y}$ and $\vec{x}$ appear as two independent space variables. As a consequence, all physical variables of the problem are, a priori, functions of both $\vec{y}$ and $\vec{x}$ :

$$
\phi=\phi(\vec{y}, \vec{x}, t) .
$$


2.2.2.2. Principle of the method: asymptotic analysis. The homogenisation method of multiple scales is based on the fundamental statement that if the scales are well separated, then all physical variables can be looked for in the form of asymptotic expansions in powers of $\varepsilon$ :

$$
\phi=\phi^{0}(\vec{y}, \vec{x}, t)+\varepsilon \phi^{1}(\vec{y}, \vec{x}, t)+\cdots,
$$

in which functions $\phi^{i}$ are $\vec{y}$-periodic.

2.2.2.3. Normalisation. The purpose of normalisation is to define and to estimate the set of nondimensional numbers that characterise the local description. The scope is to express the local description in a non-dimensional form. For estimating the non-dimensional numbers, the choice of a reference characteristic length is required. This arbitrary choice does not affect the final result. Let us consider $L$ as the reference length. The choice of the non-dimensional gradient operator is conditioned by that of the reference length. In effect, the partial derivative with respect to the physical space variable, $\vec{X}$, can be written as

$$
\frac{\partial}{\partial X_{i}}=\frac{1}{l} \frac{\partial}{\partial y_{i}}+\frac{1}{L} \frac{\partial}{\partial x_{i}} .
$$

Since $L$ is the reference characteristic length, the non-dimensional gradient operator is therefore given by

$$
\varepsilon^{-1} \vec{\nabla}_{y}+\vec{\nabla}_{x},
$$

where $\vec{\nabla}_{y}$ and $\vec{\nabla}_{x}$ are the gradient operators with respect to variables $\vec{y}$ and $\vec{x}$, respectively.

The non-dimensional numbers are estimated with respect to the small parameter $\varepsilon$. For instance, consider a given non-dimensional number $Q$. When using $L$ as the reference length, the estimation of $Q$ is denoted $Q_{L}$, and $Q_{L}$ is said to be of the order of $\varepsilon^{q}$ when

$$
\varepsilon^{q+1} \ll Q_{L} \ll \varepsilon^{q-1} .
$$

Once all non-dimensional numbers have been estimated, the local description may then be written in the form

$$
\sum_{i} \varepsilon^{i} A_{i}=0
$$

in which all operators $A_{i}$ are non-dimensional operators.

2.2.2.4. Derivation of the macroscopic description. The method consists in incorporating the asymptotic expansions of the physical variables in the non-dimensional local description. Solving the boundary-value problems arising at the successive orders of $\varepsilon$ leads to the macroscopic description. In particular, a balance equation at a given order will yield 


$$
\vec{\nabla}_{y} \cdot \phi^{i+1}+\vec{\nabla}_{x} \cdot \phi^{i}=0
$$

where $\phi^{i}$ has previously been defined while solving a boundary-value problem at a former order.

This equation expresses the balance, over the period, of the quantity $\phi^{i+1}$, in the presence of the source term $\vec{\nabla}_{x} \cdot \phi^{i}$. This is physically consistent only if the source term is average to zero. In effect, integration of (13) over the period gives

$$
\left\langle\vec{\nabla}_{x} \cdot \phi^{i}\right\rangle_{\Omega}=0
$$

in which the average over the period, $\langle\cdot\rangle_{\Omega}$, is defined by

$$
\langle\cdot\rangle_{\Omega}=\frac{1}{\Omega} \int_{\Omega} \cdot \mathrm{d} \Omega .
$$

Eq. (14) is called the 'compatibility condition' and leads to the macroscopic behaviour:

$$
\vec{\nabla}_{x} \cdot\left\langle\phi^{i}\right\rangle_{\Omega}=0
$$

\section{Homogenisation of linear viscoelastic fluid flow in porous media}

\subsection{Local description and estimations}

Consider a rigid periodic porous medium, whose period $\Omega$ is $\mathrm{O}(l)$ and characterises the porescale. Within the period, the solid and the pore spaces occupy domains $\Omega_{s}$ and $\Omega_{l}$, respectively, and their common boundary is denoted $\Gamma$ (Fig. 1). We assume the scales to be separated, i.e. the characteristic macroscopic length, $L$, is such that

$$
\varepsilon=\frac{l}{L} \ll 1
$$

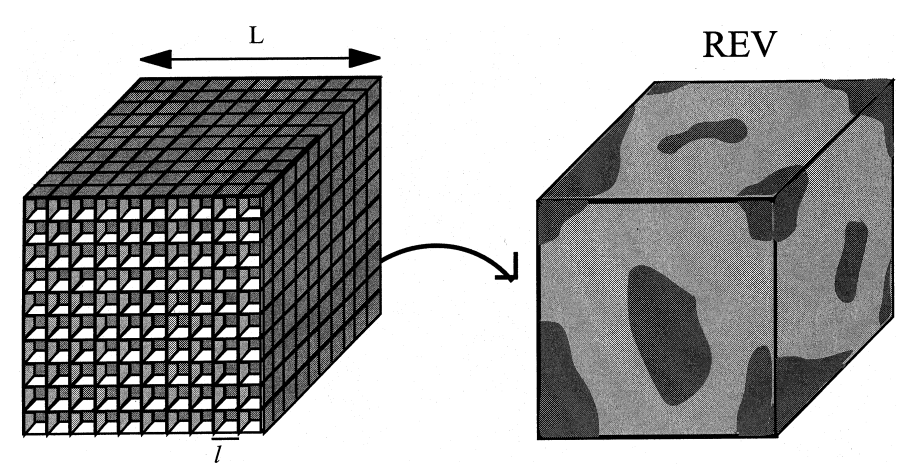

Fig. 1. Porous medium. 
The pore space is saturated by an incompressible linear viscoelastic fluid. For slow flows (i.e. for negligible Reynolds number), the momentum balance equation can be linearised:

$$
\rho \frac{\partial \vec{v}}{\partial t}=-\vec{\nabla} p+\vec{\nabla} \tilde{\tau}
$$

For a linear viscoelastic fluid, the deviatoric part of the stress tensor, $\tilde{\tau}$, is such that

$$
\left.\tilde{\tau}+\lambda_{1} \frac{\partial \tilde{\tau}}{\partial t}+\left(\lambda_{2}\right)^{2} \frac{\partial^{2} \tilde{\tau}}{\partial t^{2}}+\cdots+\left(\lambda_{n}\right)^{n} \frac{\partial^{n} \tilde{\tau}}{\partial t^{n}}=2 \mu \quad \tilde{D}+\theta_{1} \frac{\partial \tilde{D}}{\partial t}+\left(\theta_{2}\right)^{2} \frac{\partial^{2} \tilde{D}}{\partial t^{2}}+\cdots+\left(\theta_{n}\right)^{n} \frac{\partial^{n} \tilde{D}}{\partial t^{n}}\right)
$$

where the $\lambda_{k}$ 's and the $\theta_{k}$ 's, $k=1, \ldots, n$, are positive constants that represent relaxation and retardation characteristic times, respectively.

The linear viscoelastic model (18), (19) is valid if all relaxation and retardation times are small compared to the characteristic time of the local flow:

$$
\lambda_{k} \ll \frac{l}{V}, \quad \theta_{k} \ll \frac{l}{V} \quad(k=1, \ldots, n),
$$

in which $V$ is a characteristic flow velocity.

For viscoelastic fluids, the key dimensionless number is the Deborah number, De. It is defined as the ratio of the largest characteristic time of the fluid to the characteristic time of the flow:

$$
D e=\operatorname{Max}\left[\frac{\lambda_{k} V}{l}, \frac{\theta_{k} V}{l}\right] \quad(k=1, \ldots, n) .
$$

Thus, condition (20) can also be expressed as

$$
D e \ll 1 .
$$

This condition means that the distance covered by a fluid particle during any $\lambda_{k}$ or $\theta_{k}$ is very small compared to the characteristic size of the pore which is $\mathrm{O}(l)$.

Hence the linear viscoelastic model (18), (19) is valid for low Reynolds and Deborah numbers. Combining Eqs. (18) and (19) gives the local momentum balance equation:

$$
\rho \sum_{k=1}^{n}\left(\lambda_{k}\right)^{k} \frac{\partial^{k+1} \vec{v}}{\partial t^{k+1}}+\sum_{k=1}^{n}\left(\lambda_{k}\right)^{k} \frac{\partial^{k}}{\partial t^{k}}(\vec{\nabla} p)-\mu \sum_{k=1}^{n}\left(\theta_{k}\right)^{k} \frac{\partial^{k}}{\partial t^{k}}(\Delta \vec{v})+\rho \frac{\partial \vec{v}}{\partial t}=-\vec{\nabla} p+\mu \Delta \vec{v} .
$$

The mass balance equation is written as

$$
\vec{\nabla} \cdot \vec{v}=0
$$

and a no-slip condition is considered on the boundary

$$
\vec{v} /_{\Gamma}=\overrightarrow{0}
$$

Eqs. (23)-(25) constitute the local description. 
To get viscoelastic effects on the macroscopic behaviour, the relaxation and retardation characteristic times must be of the same order of magnitude as the dynamic characteristic time, i.e. the characteristic time introduced by the term $\rho \partial \vec{v} / \partial t$, which we denote by $T$. Therefore, we assume

$$
\frac{T}{\lambda_{k}}=\mathrm{O}(1), \quad \frac{T}{\theta_{k}}=\mathrm{O}(1) \quad(k=1, \ldots, n) .
$$

From the momentum balance equation arise the following dimensionless numbers:

$$
\begin{aligned}
& Q=\frac{|\vec{\nabla} p|}{|\mu \Delta \vec{v}|}, \\
& R e_{t}=\frac{|\rho \partial \vec{v} / \partial t|}{|\mu \Delta \vec{v}|}, \\
& \Lambda_{k}=|\vec{\nabla} p| /\left|\left(\lambda_{k}\right)^{k} \frac{\partial^{k}}{\partial t^{k}}(\overrightarrow{\nabla p})\right| \quad(k=1, \ldots, n), \\
& \Theta_{k}=|\mu \Delta \vec{v}| /\left|\mu\left(\theta_{k}\right)^{k} \frac{\partial^{k}}{\partial t^{k}}(\Delta \vec{v})\right| \quad(k=1, \ldots, n) .
\end{aligned}
$$

Let us choose $L$ as the reference characteristic length.

The order of magnitude of $Q_{L}$ is given by the physics. In effect, the local flow, characterised by the term $\mu \Delta \vec{v}$ is due to the macroscopic pressure gradient. As a result, we have

$$
\mu \frac{V}{l^{2}}=\mathrm{O}\left(\frac{\delta P}{L}\right) .
$$

$\delta P$ is the characteristic increment of pressure over the distance $L$.

Thus, we deduce

$$
Q_{L}=\frac{|\vec{\nabla} p|_{L}}{|\mu \Delta \vec{v}|_{L}}=\frac{\delta P / L}{\mu V / L^{2}}=\mathrm{O}\left(\varepsilon^{-2}\right) .
$$

Now, in order to get macroscopic transient effects, the transient term, $\rho \partial \vec{v} / \partial t$, must be locally, i.e. with respect to the microscopic characteristic length, $l$, of the same order of magnitude as the viscous term, $\mu \Delta \vec{v}$ : 


$$
|\mu \Delta \vec{v}|_{l}=\left|\rho \frac{\partial \vec{v}}{\partial t}\right|_{l},
$$

from which we deduce the order of magnitude of the transient Reynolds number:

$$
\operatorname{Re}_{t_{L}}=\frac{|\rho \partial \vec{v} / \partial t|_{L}}{|\mu \Delta \vec{v}|_{L}}=\frac{|\rho \partial \vec{v} / \partial t|_{l}}{|\mu \Delta \vec{v}|_{l}} \cdot \frac{l^{2}}{L^{2}}=\mathrm{O}\left(\varepsilon^{-2}\right) .
$$

Finally, according to (26), we deduce

$$
\begin{aligned}
& \Lambda_{k_{L}}=\mathrm{O}\left(\left(\frac{T}{\lambda_{k}}\right)^{k}\right)=\mathrm{O}(1), \\
& \Theta_{k_{L}}=\mathrm{O}\left(\left(\frac{T}{\theta_{k}}\right)^{k}\right)=\mathrm{O}(1) .
\end{aligned}
$$

The normalised local description is the following:

$$
\begin{aligned}
& \rho \sum_{k=1}^{n}\left(\lambda_{k}\right)^{k} \frac{\partial^{k+1} \vec{v}}{\partial t^{k+1}}+\sum_{k=1}^{n}\left(\lambda_{k}\right)^{k} \frac{\partial^{k}}{\partial t^{k}}(\vec{\nabla} p)-\varepsilon^{2} \mu \sum_{k=1}^{n}\left(\theta_{k}\right)^{k} \frac{\partial^{k}}{\partial t^{k}}(\Delta \vec{v})+\rho \frac{\partial \vec{v}}{\partial t}=-\vec{\nabla} p+\varepsilon^{2} \mu \Delta \vec{v}, \\
& \vec{\nabla} \cdot \vec{v}=0, \\
& \vec{v} / \Gamma=\overrightarrow{0},
\end{aligned}
$$

in which all quantities are now dimensionless.

In Fourier space the normalised local description is written as

$$
\begin{aligned}
& \tilde{\rho} \vec{v}=-\overrightarrow{\nabla p}+\varepsilon^{2} \tilde{\mu} \Delta \vec{v}, \\
& \vec{\nabla} \cdot \vec{v}=0, \\
& \vec{v} /_{\Gamma}=\overrightarrow{0},
\end{aligned}
$$

where

$$
\begin{aligned}
& \tilde{\rho}=\mathrm{i} \omega \rho, \\
& \tilde{\mu}=\mu S(\mathrm{i}, \omega),
\end{aligned}
$$

and

$$
S(\mathrm{i}, \omega)=\frac{1+\theta_{1} \mathrm{i} \omega+\left(\theta_{2} \mathrm{i} \omega\right)^{2}+\cdots+\left(\theta_{n} \mathrm{i} \omega\right)^{n}}{1+\lambda_{1} \mathrm{i} \omega+\left(\lambda_{2} \mathrm{i} \omega\right)^{2}+\cdots+\left(\lambda_{n} \mathrm{i} \omega\right)^{n}} .
$$




\subsection{Derivation of the macroscopic description}

We now introduce the multiple scale coordinates $\vec{y}$ and $\vec{x}$ and the perturbation expansions

$$
\begin{aligned}
& p(\vec{y}, \vec{x}, t)=p^{0}(\vec{y}, \vec{x}, t)+\varepsilon p^{1}(\vec{y}, \vec{x}, t)+\ldots, \\
& \vec{v}(\vec{y}, \vec{x}, t)=\vec{v}^{0}(\vec{y}, \vec{x}, t)+\varepsilon \vec{v}^{1}(\vec{y}, \vec{x}, t)+\ldots,
\end{aligned}
$$

where $\vec{v}^{i}$ and $p^{i}$ are $\vec{y}$-periodic.

Incorporating expansions (46) and (47) in (40) leads at the two first orders $\mathrm{O}\left(\varepsilon^{-1}\right)$ and $\mathrm{O}\left(\varepsilon^{0}\right)$ :

$$
\begin{aligned}
& \vec{\nabla}_{y} p^{0}=\overrightarrow{0}, \\
& \tilde{\rho} \vec{v}^{0}=\tilde{\mu} \Delta_{y} \vec{v}^{0} \nabla_{y} p^{1}-\vec{\nabla}_{x} p^{0} .
\end{aligned}
$$

Similarly, from (41), we get

$$
\begin{aligned}
& \vec{\nabla}_{y} \cdot \vec{v}^{0}=0, \\
& \vec{\nabla}_{y} \vec{v}^{1}+\vec{\nabla}_{x} \vec{v}^{0}=0 .
\end{aligned}
$$

Finally, boundary-condition (42) gives

$$
\vec{v}^{0} /{ }_{\Gamma}=\vec{v}^{1} /{ }_{\Gamma}=\cdots=\overrightarrow{0} .
$$

The method consists now in deducing the boundary-value problems at successive orders and in solving them over the period.

First-order problem.

$$
\vec{\nabla}_{y} p^{0}=\overrightarrow{0}
$$

It gives

$$
p^{0}=p^{0}(\vec{x}, t)
$$

Second-order problem.

$$
\begin{aligned}
& \tilde{\rho} \vec{v}^{0}=\tilde{\mu} \Delta_{y} \vec{v}^{0}-\nabla_{y} p^{1}-\vec{\nabla}_{x} p^{0}, \\
& \vec{\nabla}_{y} \cdot \vec{v}^{0}=0, \\
& \vec{v}^{0} /_{\Gamma}=\overrightarrow{0},
\end{aligned}
$$

$\left(\vec{v}^{0}\right.$ and $p^{1}$ are $\vec{y}$-periodic).

Let $\mathscr{V}(\Omega)$ be the Hilbert space of $\Omega$-periodic and complex-valued vectors $\vec{\alpha}$, that are defined over $\Omega_{l}$ and such that: $\vec{\nabla}_{y} \cdot \vec{\alpha}=0$ and $\left.\vec{\alpha}\right|_{\Gamma}=\overrightarrow{0} . \mathscr{V}(\Omega)$ is equipped with the following inner product: 


$$
\left.(\vec{\alpha}, \vec{\beta})_{\mathscr{r}(\Omega)}=\int_{\Omega_{l}} \frac{\partial \alpha_{i}}{\partial y_{j}} \frac{\partial \tilde{\beta}_{i}}{\partial y_{j}}+\alpha_{i} \tilde{\beta}_{i}\right) \mathrm{d} \Omega,
$$

where $\tilde{\vec{\beta}}$ is the conjugate of $\vec{\beta}$.

The weak formulation of (55)-(57) is given by

$$
\forall \vec{\alpha} \in \mathscr{V}(\Omega): a\left(\vec{v}^{0}, \vec{\alpha}\right)_{\mathscr{V}(\Omega)}=-\int_{\Omega_{l}} \frac{\partial p^{0}}{\partial x_{i}} \tilde{\alpha}_{i} \mathrm{~d} \Omega,
$$

where $a(\vec{\alpha}, \vec{\beta})_{\mathscr{V}(\Omega)}$ is a sesquilinear form and is defined by

$$
\left.a(\vec{\alpha}, \vec{\beta})_{\mathscr{V}(\Omega)}=\int_{\Omega_{l}} \tilde{\mu} \frac{\partial \alpha_{i}}{\partial y_{j}} \frac{\partial \tilde{\beta}_{i}}{\partial y_{j}}+\tilde{\rho} \alpha_{i} \tilde{\beta}_{i}\right) \mathrm{d} \Omega .
$$

It can be shown that $\operatorname{Re}\left(a(\vec{\alpha}, \vec{\alpha})_{\mathscr{r}(\Omega)}\right)>0$. Hence, by Lax-Milgram Lemma, there is a unique solution to (59). Let $\vec{k}^{j}$ be the particular solution of (59) for $\partial p^{0} / \partial x_{i}=\delta_{i j}$ ( $j$ fixed). Thus, $\vec{v}^{0}$ is written as

$$
\vec{v}^{0}=-\tilde{k}(\vec{y}, \omega) \vec{\nabla}_{x} p^{0} .
$$

Third-order problem.

$$
\begin{aligned}
& \vec{\nabla}_{y} \vec{v}^{1}+\vec{\nabla}_{x} \vec{v}^{0}=0, \\
& \vec{v}^{1} /_{\Gamma}=\overrightarrow{0} .
\end{aligned}
$$

Integrating (62) over $\Omega$ and then using the divergence theorem, boundary condition (63) and the condition of periodicity leads to the macroscopic description:

$$
\vec{\nabla}_{x} \cdot\left\langle\vec{v}^{0}\right\rangle_{\Omega}=0
$$

in which

$$
\left\langle\vec{v}^{0}\right\rangle_{\Omega}=-\tilde{K}(\omega) \vec{\nabla}_{x} p^{0}
$$

where

$$
\tilde{K}=\langle\tilde{k}\rangle_{\Omega}, \quad\langle\cdot\rangle_{\Omega}=\frac{1}{|\Omega|} \int_{\Omega_{l}} \cdot \mathrm{d} \Omega .
$$

Eq. (65) is the generalised Darcy's law [7]. $\tilde{K}$ is a $\omega$ dependent, complex-valued second order tensor and is called the 'dynamic permeability'. When $\omega=0, \tilde{K}$ is the intrinsic permeability. $\tilde{K}$ is a symmetrical tensor; its real part is definite positive whereas its imaginary part is definite negative. As a result, $\tilde{K}$ is inversible. Let $\tilde{H}(\omega)$ be the inverse tensor of $\tilde{K}$ : 


$$
\tilde{H}(\omega)=\tilde{K}^{-1}(\omega)=\tilde{H}_{r}+\mathrm{i} \tilde{H}_{i},
$$

$\tilde{H}(\omega)$ is the dynamic resistivity.

In terms of the dynamic resistivity, the filtration law (65) is expressed as follows:

$$
\vec{\nabla}_{x} p^{0}=-\tilde{H}(\omega)\left\langle\vec{v}^{0}\right\rangle_{\Omega}
$$

or

$$
\vec{\nabla}_{x}\left(n p^{0}\right)=-n \tilde{H}_{r}(\omega)\left\langle\vec{v}^{0}\right\rangle_{\Omega}-n \frac{\tilde{H}_{i}(\omega)}{\omega} \frac{\partial}{\partial t}\left(\left\langle\vec{v}^{0}\right\rangle_{\Omega}\right)
$$

where

$$
n=\frac{\left|\Omega_{l}\right|}{|\Omega|}
$$

is the porosity.

At constant pulsation, Eq. (69) is a real-valued Darcy's law. Written in the above form, the seepage law expresses a momentum balance: the partial pressure $n p^{0}$ is the fluid stress, the term $n \tilde{H}_{r}(\omega)$ is related to the viscous dissipation, and $n \tilde{H}_{i}(\omega) / \omega$ is the apparent density of the fluid in the porous medium.

\section{Results for the filtration of an Oldroyd fluid in a bundle of capillary tubes}

The purpose of this section is to examine the macroscopic filtration law obtained for the flow of an Oldroyd fluid in a bundle of capillary tubes (Fig. 2).

The model of Oldroyd is a simplification of the generalised linear viscoelastic model (19):

$$
\left.\tilde{\tau}+\lambda \frac{\partial \tilde{\tau}}{\partial t}=2 \mu \quad \tilde{D}+\theta \frac{\partial \tilde{D}}{\partial t}\right)
$$

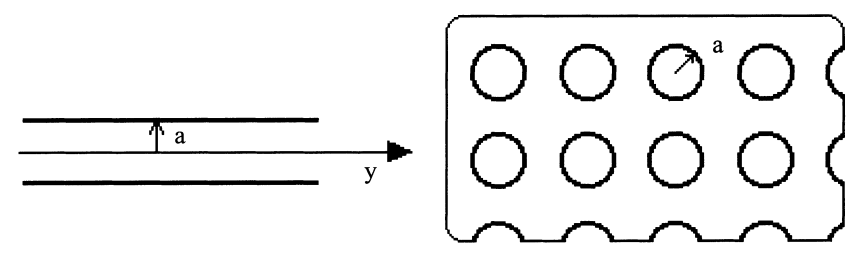

(a)

(b)

Fig. 2. Bundle of capillary tubes: (a) capillary tube with respect to variable $\vec{y}$; (b) cross-section of the medium with respect to variable $\vec{x}$. 
in which $\lambda$ and $\theta$ are the relaxation and the retardation characteristic times, respectively. In Fourier space, model (70) can also be expressed as follows:

$$
\tilde{\tau}=2 \mu S \tilde{D}, \quad S=\frac{1+\mathrm{i} \omega \theta}{1+\mathrm{i} \omega \lambda} .
$$

When $\lambda=\theta$, relation (70) represents a viscous Newtonian fluid, whereas $\theta=0, \lambda \neq 0$ characterises a Maxwellian fluid. If we assume, according to Biot $[8,11,12]$ that there exist hidden variables and that the model may be expressed in Lagrangian form

$$
\tau_{i j}=\frac{\partial \mathscr{W}}{\partial \varepsilon_{i j}}+\frac{\partial \mathscr{D}}{\partial D_{i j}}
$$

where $\tilde{\varepsilon}$ is the deformation, $\mathscr{W}$ the potential energy and $\mathscr{D}$ the dissipation, then, we obtain

$$
\tau_{i j}=\mu \omega^{2} \frac{\lambda-\theta}{1+\omega^{2} \lambda^{2}} \tilde{\varepsilon}+\mu \frac{1+\omega^{2} \lambda \theta}{1+\omega^{2} \lambda^{2}} \tilde{D} .
$$

Thus, assuming the potential energy being positive semi-definite yields

$$
\lambda-\theta \geqslant 0 .
$$

Models made of dashpots and springs do verify this condition. Other investigations based on the kinetic theory of dilute solution of elastic dumbbels also yield to condition (74) (see [13]). However, there is no convincing argument that it should be applied to actual materials. Therefore, for the sake of generality, we disregard it in the following.

According to the results obtained in Section 3, the macroscopic filtration law of the flow of an Oldroyd fluid in a bundle of capillary tubes of radius $a$ is described by

$$
\left\langle v^{0}\right\rangle_{\Omega}=-K(\omega) \frac{\mathrm{d} p_{0}}{\mathrm{~d} x},
$$

where the dynamic permeability, $K(\omega)$, is

$$
K=\frac{n}{\pi a^{2}} \int_{0}^{a} 2 \pi r k(r, \omega) \mathrm{d} r,
$$

in which cylindrical coordinates $(r, \theta, x)$ are used. $\tilde{k}$ is such that $v^{0}=-\tilde{k} \mathrm{~d} p^{0} / \mathrm{d} x$ is the solution of the local problem (55)-(57), which in a single capillary tube is expressed as

$$
\begin{aligned}
& \tilde{\mu}\left(\frac{\mathrm{d}^{2} v^{0}}{\mathrm{~d} r^{2}}+\frac{1}{r} \frac{\mathrm{d} v^{0}}{\mathrm{~d} r}\right)-\tilde{\rho} v^{0}=\frac{\mathrm{d} p^{0}}{\mathrm{~d} x}, \\
& v^{0}(a)=0,
\end{aligned}
$$


where

$$
\tilde{\rho}=\mathrm{i} \omega \rho, \quad \tilde{\mu}=\mu S, \quad \tilde{v}=\frac{\tilde{\mu}}{\rho}, \quad S=\frac{1+\mathrm{i} \omega \theta}{1+\mathrm{i} \omega \lambda} .
$$

The solution of (77) and (78) is

$$
v^{0}=-k(r, \omega) \frac{\mathrm{d} p^{0}}{\mathrm{~d} x}, \quad k=\frac{1}{\tilde{\rho}}\left(1-\frac{J_{0}\left(\mathrm{i} \sqrt{\mathrm{i} \omega \tilde{v}^{-1}} r\right)}{J_{0}\left(\mathrm{i} \sqrt{\mathrm{i} \omega \tilde{v}^{-1}} a\right)}\right),
$$

from which we deduce the expression for the dynamic permeability $K(\omega)$ :

$$
K=-\frac{n}{\tilde{\rho}} \frac{J_{2}\left(\mathrm{i} \sqrt{\mathrm{i} \omega \tilde{v}^{-1}} a\right)}{J_{0}\left(\mathrm{i} \sqrt{\mathrm{i} \omega \tilde{v}^{-1}} a\right)} .
$$

$J_{2}$ and $J_{0}$ are the Bessel functions.

Now let us express seepage law (75) in the form of a momentum balance (as in (69):

$$
\begin{aligned}
& -\nabla n p^{0}=-n H\left\langle v^{0}\right\rangle=-n H_{r}\left\langle v^{0}\right\rangle-\frac{n H_{i}}{\omega} \frac{\mathrm{d}\left\langle v^{0}\right\rangle}{\mathrm{d} t}, \\
& H=H_{r}+\mathrm{i} H_{i}=K^{-1} .
\end{aligned}
$$

Low frequencies. For low frequencies, it is easy to show that the behaviour is given by

$$
n H_{r}=\frac{8 \mu}{a^{2}}, \quad \frac{n H_{i}}{\omega}=\rho\left(\frac{4}{3}+\frac{8 v}{a^{2}}(\theta-\lambda)\right) .
$$

As expected, the dissipation term, $n H_{r}$, is that of a Newtonian fluid of viscosity $\mu$.

For a Newtonian fluid, i.e. for $\lambda=\theta$, we retrieve the following result for the apparent density,

$$
\frac{n H_{i}}{\omega}=\frac{4}{3} \rho,
$$

that characterises the added mass density as shown by Biot, [9]. Furthermore, we see that the apparent density becomes negative when

$$
\lambda-\theta>\frac{a^{2}}{6 v} .
$$

High frequencies. For high frequencies, the apparent density tends to the fluid density when $\lambda \neq 0$,

$$
\lim _{\omega \rightarrow \infty} \frac{n H_{i}}{\omega}=\rho,
$$

and it tends to a constant which is different from $\rho$ when $\lambda=0$. 
As for the dissipation, two different behaviours appear when the frequency is high:

$$
\begin{array}{ll}
\lim _{\omega \rightarrow \infty} n H_{r}=\text { constant } & \text { if } \lambda \neq 0 \text { and } \theta=0 \text { or if } \lambda=0 \text { and } \theta \neq 0, \\
\lim _{\omega \rightarrow \infty} n H_{r} \sim \sqrt{\omega} & \text { otherwise. }
\end{array}
$$

General case. In general cases, five distinct behaviours for the monochromatic seepage law may be distinguished.

1. Newtonian fluids, $\lambda=\theta . K$ and $H$ are shown in Fig. 3. This is the classical case of the dynamics of a Newtonian fluid in a porous medium which has been investigated by Biot, [10], and revisited in $[14,7]$ from the homogenisation point of view. The behaviour shows an added mass density for small values of $\omega$.

2. Maxwell fluids, $\theta=0, \lambda \neq 0$. This case has already been investigated by volume averaging in [15]. $K$ and $H$ are shown in Fig. 4. Note the negative apparent density for small values of $\omega$.

3. Second order fluid with vanishing second normal stress coefficient, i.e. Oldroyd fluid with $\theta \neq 0, \lambda=0$. The curves are presented in Fig. 5. Both the apparent density and the dissipation are independent of the frequency. Condition (74) is not checked in this case.

4. Oldroyd fluid with $\lambda \neq 0, \theta \neq 0, \lambda-\theta>0$. The apparent density is negative at low frequencies and the dissipation presents a minimum (see Fig. 6).

5. Oldroyd fluid with $\lambda \neq 0, \theta \neq 0, \lambda-\theta<0$. The behaviour of $H$ is similar to that obtained for a Newtonian fluid $(\lambda=\theta)$. However $K$ and $H$ show an additional plateau (see Fig. 7). Condition (74) is not checked in this case.

Comparison with the phenomenological model (2). Let us consider the phenomenological model of [3] that has been mentioned in the introduction (see Eq. (2)). This model is built by analogy
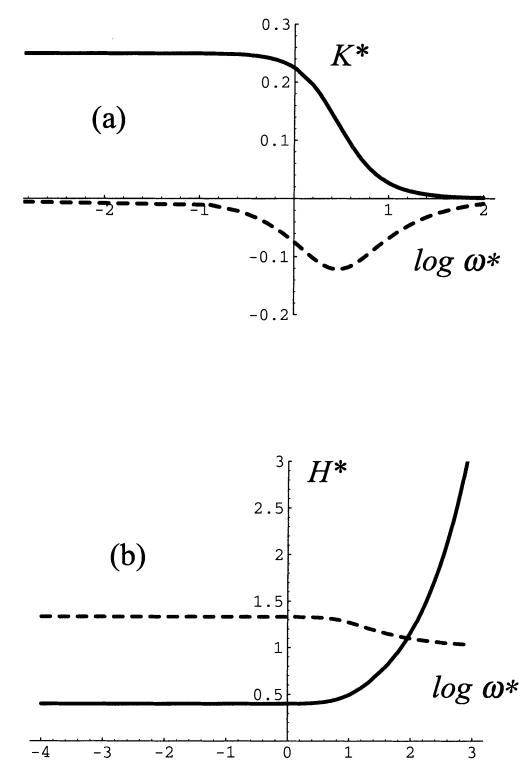

Fig. 3. Newtonian fluid: (a) dynamic permeability: bold: $K_{r}^{*}=2 \mu K_{r} / n a^{2}$, dashed: $K_{i}^{*}=2 \mu K_{i} / n a^{2}$; (b) dynamic resistivity: bold: $H_{r}^{*}=0.1 n a^{2} H_{r} / 2 \mu$, dashed: $H_{i}^{*}=n H_{i} / \rho \omega$, dimensionless frequency: $\omega^{*}=\omega a^{2} / 2 v$. 

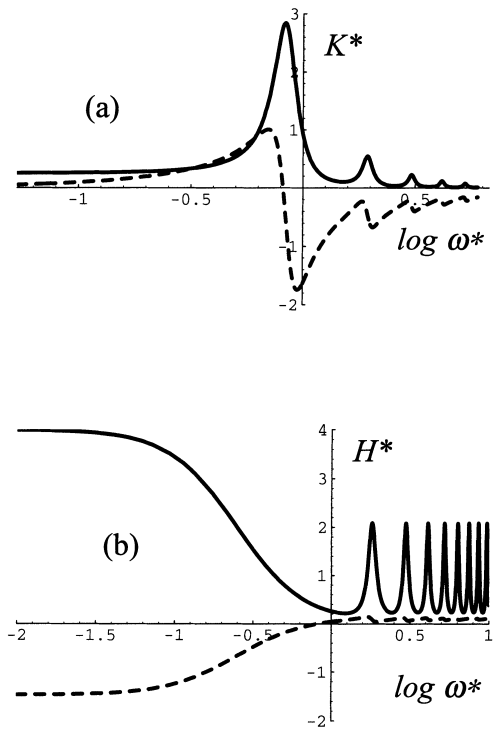

Fig. 4. Maxwell fluid, $\lambda=0.02$. As in Fig. 3, now with $H_{r}^{*}=n a^{2} H_{r} / 2 \mu, H_{i}^{*}=0.1 n H_{i} / \rho \omega$.
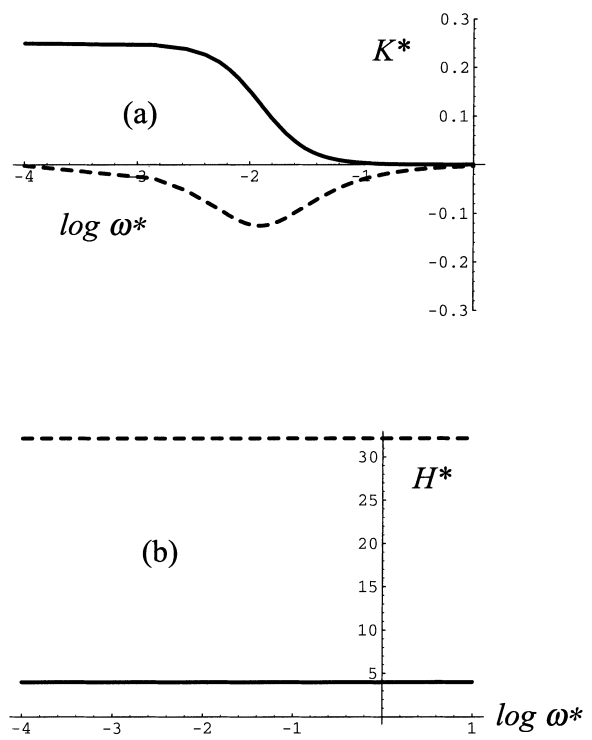

Fig. 5. Oldroyd fluid, $\lambda=0, \theta=0.4$. As in Fig. 3, now with $H_{r}^{*}=n a^{2} H_{r} / 2 \mu, H_{i}^{*}=0.1 n H_{i} / \rho \omega$.

with the Oldroyd fluid model as an attempt to describe the filtration of an Oldroyd fluid in a porous medium. In Fourier space it is written as

$$
\left(1+\mathrm{i} \omega \lambda_{v}\right) \vec{v}=-\frac{k}{\mu}\left(1+\mathrm{i} \omega \lambda_{p}\right) \vec{\nabla} p
$$



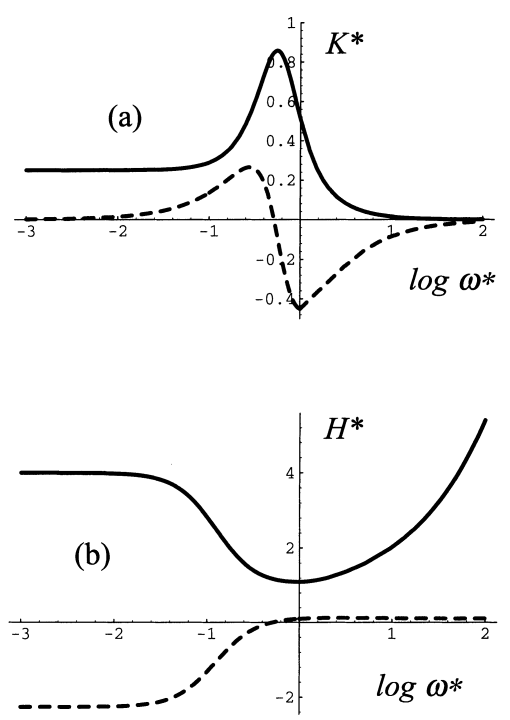

Fig. 6. Oldroyd fluid, $\lambda>\theta \neq 0 . \quad$ As in Fig. 3, now with $H_{r}^{*}=n a^{2} H_{r} / 2 \mu, \quad H_{i}^{*}=0 . \ln H_{i} / \rho \omega$ $\left(\lambda=0.04, \theta=0.01, v=10^{-4} \mathrm{~m}^{2} / \mathrm{s}, a=10^{-3} \mathrm{~m}\right)$.
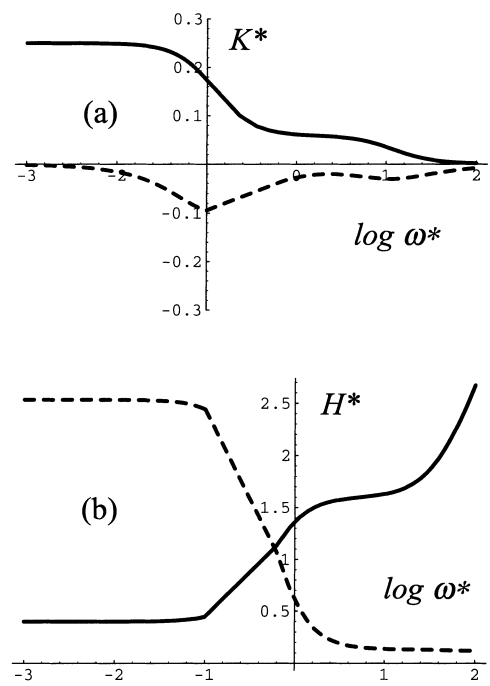

Fig. 7. Oldroyd fluid, $\theta>\lambda \neq 0 . \quad$ As in Fig. 3, now with $H_{r}^{*}=n a^{2} H_{r} / 2 \mu, \quad H_{i}^{*}=0.1 n H_{i} / \rho \omega$ $\left(\lambda=0.01, \theta=0.04, v=10^{-4} \mathrm{~m}^{2} / \mathrm{s}, a=10^{-3} \mathrm{~m}\right)$.

from which we define a phenomenological dynamic permeability, $K_{\mathrm{ph}}$ and the corresponding phenomenological dynamic resistivity:

$$
K_{\mathrm{ph}}=\frac{k}{\mu} \frac{1+\mathrm{i} \omega \lambda_{p}}{1+\mathrm{i} \omega \lambda_{v}}, \quad H_{p h}=\frac{1}{K_{p h}} .
$$



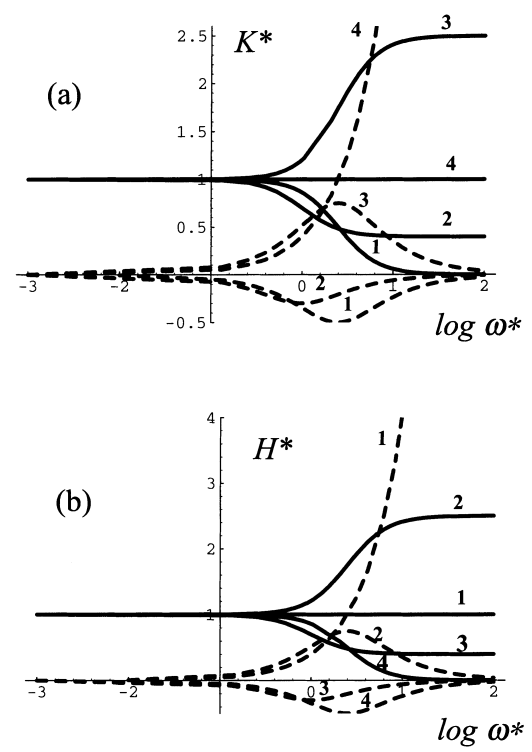

Fig. 8. Phenomenological model: (a) dynamic permeability: bold: $K_{r}^{*}=K_{r}^{\mathrm{ph}} / k_{0}$, dashed: $K_{i}^{*}=K_{i}^{\mathrm{ph}} / k_{0}$; (b) dynamic resistivity: bold: $H_{r}^{*}=H_{r}^{\mathrm{ph}} / k_{0}$, dashed: $H_{i}^{*}=H_{r}^{\mathrm{ph}} / k_{0}, 1: \lambda_{p}=0, \lambda_{v}=0.4,2: \lambda_{p}=0.4, \lambda_{v}=1,3: \lambda_{p}=1, \lambda_{v}=0.4,4$ : $\lambda_{p}=0.4, \lambda_{v}=0$.

The main profiles of $K_{\mathrm{ph}}$ and $H_{\mathrm{ph}}$ are shown in Fig. 8. It is clear that these results strongly differ from those obtained via homogenisation. The analysis is easier by comparing dynamic resistivities rather than dynamic permeabilities, as physically, $H$ is a more meaningful quantity. We see that the profile of $H$ approaches the Newtonian fluid behaviour, only, (Fig. 8 case 1), with the restriction that the apparent density remains constant whereas it varies with respect to $\omega$ for a Newtonian fluid.

\section{Conclusions}

We have used the homogenisation method so as to derive a general filtration law describing the flow of a linear viscoelastic fluid in a porous medium. This model is valid under quite restrictive conditions as in particular the Reynolds and Deborah numbers must be low. Anyhow this model is rigorous within its domain of validity. It is expressed either as a generalised Darcy's law (65) with a dynamic permeability tensor $\tilde{K}(\omega)$, or as a momentum balance (68) in terms of the dynamic resistivity tensor $\tilde{H}(\omega)=K^{-1}$.

The results obtained in the particular case of the flow of an Oldroyd fluid in a bundle of capillary tubes show that the viscoelastic behaviour strongly differs from the Newtonian behaviour. It also shows that negative apparent densities can be obtained. Finally, comparison of these results with those obtained for model (2) shows that the question of an appropriate phenomenological filtration law for describing the flow of an Oldroyd fluid in a porous medium is still open. 


\section{Acknowledgements}

Part of this work has been performed while B. Khuzhayorov was on a two month leave at the "Laboratoire Sols, Solides, Structures" in Grenoble (France). The French Ambassy in Tashkent (Uzbekistan) is greatly acknowledged for the financial support of this fruitful cooperation.

J.-L. Auriault wishes to thank Mobil Oil Technology Company for financial support via a research gift.

The authors are grateful to C. Verdier from "Laboratoire de Rhéologie" in Grenoble, for his valuable advice.

\section{References}

[1] L. Skartsis, B. Khomami, J.L. Kardos, Polymeric flow through fibrous media, J. Rheology 36 (4) (1992) $589-620$.

[2] M.G. Alishayev, Proceedings of moscow pedagogy institute (in Russian), Hydromechanics 3 (1974) $166-174$.

[3] M.G. Alishayev, A.Kh. Mirzadzhanzadeh, About retardation phenomena in filtration theory (in Russian), Nieft i gaz 6 (1975) 71-74.

[4] A. Bensoussan, J.L. Lions, G. Papanicolaou, Asymptotic Analysis for Periodic Structures, North-Holland, Amsterdam, 1978.

[5] E. Sanchez-Palencia, Non-homogeneous Media and Vibration Theory, Lecture Notes in Physics, vol. 127, Springer, Berlin, 1980.

[6] J.-L. Auriaul, Heterogeneous medium: Is an equivalent description possible? Int. J. Eng. Sci. 29 (1991) $785-795$.

[7] J.-L. Auriault, Dynamic behaviour of a porous medium saturated by a newtonian fluid, Int. J. Eng. Sc. 18 (1980) $775-785$.

[8] M.A. Biot, Theory of stress-strain relations in anisotropic viscoelasticity and relaxation phenomena, J. Appl. Phys. 25 (11) (1954) 1385-1391.

[9] M.A. Biot, Theory of propagation of elastic waves in a fluid-saturated porous solid. I Low-frequency range, J.A.S.A. 28 (2) (1956) 168-178.

[10] M.A. Biot, Theory of propagation of elastic waves in a fluid-saturated porous solid. II Higher-frequency range, J.A.S.A. 28 (2) (1956) 179-191.

[11] M.A. Biot, Linear thermodynamics and the mechanics of solids, in: Proceedings of the Third US National Congress on Applied Mechanics, New-York, 1958, pp. 1-18.

[12] M.A. Biot, Mechanics of Incremental Deformation, Wiley, New York, 1965.

[13] R.B. Bird, C.F. Curtiss, R.C. Armstrong, O. Hassager, Dynamics of Polymeric Liquids, vol. 2, Wiley, New York, 1987.

[14] T. Levy, Propagation of waves in a fluid saturated porous elastic solid, Int. J. Eng. Sc. 17 (1979) $1005-1014$.

[15] M. López De Haro, J.A. Del Río, S. Whitaker, Flow of Maxwell fluids in porous media, Transport in Porous Media 25 (2) (1996) 167-192. 